\title{
Comparative evaluation of the new Sheffield table and the modified joint British societies coronary risk prediction chart against a laboratory based risk score calculation
}

\author{
K S Rabindranath, N R Anderson, R Gama, M R Holland
}

Postgrad Med J 2002;78:269-272

See end of article for authors' affiliations

Correspondence to: Dr R Gama, Clinical Chemistry, New Cross Hospital, Wolverhampton, West Midlands

WVIO OQP, UK;

dr.gama@rwh-tr.nhs.uk

Submitted

20 November 2001

Accepted

20 February 2002

Background: Management of borderline hypertension and hypercholesterolaemia is based on an individual's coronary heart disease (CHD) risk rather than arbitrary values for blood pressure or serum cholesterol. Prediction of CHD risk involves using tables, charts, or computer programs based on the Framingham equations. The new Sheffield table and modified joint British societies coronary risk prediction (JBS) chart are widely used. The JBS chart approximates age and systolic blood pressure, and the new Sheffield table dichotomises blood pressure, and these simplifications may lead to diagnostic inaccuracy.

Methods: The diagnostic performance of the charts against an individualised laboratory based CHD risk calculation in 1102 subjects in primary care were evaluated and compared.

Results: The new Sheffield table and modified JBS chart performed equally well with a respective diagnostic sensitivity and specificity of $91.6 \%(95 \%$ confidence interval $86.7 \%$ to $95.1 \%)$ and $93.8 \%$ $(91.1 \%$ to $97.9 \%)$, and $93.6 \%(90.4 \%$ to $96.0 \%)$ and $94.7 \%(92.6 \%$ to $96.1 \%)$ at 10 year CHD risk of $15 \%$; and of $95.2 \%(82.8 \%$ to $99.4 \%)$ and $97.9 \%(96.8 \%$ to $98.7 \%)$, and $90.5 \%(75.6 \%$ to $97.4 \%)$ and $100 \%(99.7 \%$ to $100 \%)$ at 10 year CHD risk of $30 \%$.

The modified JBS chart graphic display provides graded risk, which may be an advantage over the new Sheffield table, which identifies thresholds of risk. The new Sheffield table, unlike any other method, can be used as screening tool for cholesterol measurement.

Conclusions: The new Sheffield table and modified JBS chart are valid for use in primary care since their diagnostic accuracy is unaffected by approximations in age and blood pressure. It is suggested that practitioners should choose whichever risk assessment tool they are comfortable with and use it.

$\mathrm{R}$ ecommendations on the management of hypercholesterolaemia, borderline hypertension, and use of aspirin in the primary prevention of coronary heart disease (CHD) are now based on an individual's absolute CHD risk rather than arbitrary values for blood pressure and serum cholesterol concentrations. ${ }^{1}$

Coronary risk assessment methods are based on the Framingham Heart Study, ${ }^{2}$ which have been validated for the British population. ${ }^{3}$ This observational study has documented population characteristics in over 10000 residents of Framingham, Massachusetts, together with causes of death for over 50 years. ${ }^{4}$ From these data, risk factors for CHD have been identified and quantified as multiple logistic equations. The Framingham equations predict CHD risk using eight weighted risk factors: age, gender, blood pressure, serum total and high density lipoprotein (HDL) cholesterol, and the presence or absence of diabetes mellitus, smoking, and electrocardiographic evidence of left ventricular hypertrophy (LVH).

An individual's CHD risk prediction usually involves referral to tables and charts or use of computer programs. ${ }^{1}$ The approximations of risk factors required to construct tables and charts may, however, affect their accuracy. The new Sheffield table and the modified joint British societies coronary prediction risk (JBS) chart are widely used. ${ }^{56}$ Apart from design and presentation, the major differences between the new Sheffield table and modified JBS chart are their representation of age, hypertension, and LVH as risk factors. The new Sheffield table represents hypertension as a dichotomous variable (that is absent or present), age (for men 26-70 years; women 36-70 years) is represented at two yearly intervals, and it corrects for
LVH by adding 20 years. ${ }^{5}$ The modified JBS chart represents hypertension as a continuous variable (systolic blood pressure $110-190 \mathrm{~mm} \mathrm{Hg}$ at $10 \mathrm{~mm} \mathrm{Hg}$ intervals), age (35-74 years) is represented at four 10 year intervals, and does not correct for LVH since the authors consider these patients to already be at high cardiovascular risk. ${ }^{6}$ It is therefore possible that these differences could influence their diagnostic accuracy and comparative diagnostic performance.

The National Service Framework on CHD recommends the modified JBS chart as the preferred method for predicting CHD risk. ${ }^{1}$ Indeed, a recent smaller study has reported that in comparison with other charts the modified JBS chart has the best sensitivity and specificity for the assessing CHD risk in primary care, ${ }^{7}$ but these results remain to be confirmed.

We have been providing general practitioners with a 10 year CHD individualised laboratory based risk score calculation based on the full Framingham equations. We used this precise laboratory calculated CHD risk to evaluate and compare the diagnostic performance of the new Sheffield table and modified JBS chart for a large group of subjects in primary care.

\section{PATIENTS AND METHODS}

Data were collected from inception of the programme on 1 September 1999 to 31 November 2000. Patients were selected

Abbreviations: $\mathrm{CHD}$, coronary heart disease; $\mathrm{HDL}$, high density lipoprotein; JBS chart, joint British societies coronary risk prediction chart; $\mathrm{LVH}$, left ventricular hypertrophy 
at the discretion of their own general practitioners. Information on age, sex, systolic blood pressure, and the presence or absence of smoking, diabetes mellitus, and LVH were collected from a self adhesive sticker fixed on the pathology request form. Serum total and HDL cholesterol were measured by routine automated kit methods (DiaSys Diagnostic Systems GmbH \& Co, Holzheim, Germany and Bio-Stat Diagnostic Systems, Stockport, UK respectively). These data were used to generate each patient's CHD risk using a computer program written by MRH based on the full Framingham equation for the laboratory computer system (LMX, Bayer Diagnostics Ltd, Newbury, UK). The results were reported as a percentage 10 year CHD risk together with a comment on modifying risk if appropriate (for example, stopping smoking) and treatment with aspirin, antihypertensives, and statins based on previous recommendations. ${ }^{18}$

Two doctors were provided with each patient's relevant clinical details, serum cholesterol, HDL cholesterol, and total: HDL cholesterol ratio to predict CHD risk using the new Sheffield table and the modified JBS risk chart. They then recorded the 10 year CHD risk as either $<15 \%, \geqslant 15$ to $<30 \%$, or $\geqslant 30 \%$. Charts were evaluated at $15 \%$ and $30 \%$ risk as these values influence the use of antihypertensives and aspirin (15\% risk) and antihyperlipidaemics (30\% risk). ${ }^{18}$

Diagnostic sensitivity, specificity, and predictive values for the modified JBS chart and the new Sheffield table were calculated using the precise individualised laboratory based risk score calculated from the full Framingham equations as the reference method. The per cent confidence interval for each value was calculated using the exact Poisson method. For statistical analysis CHD risk scores of $\leqslant 14.9$ were assigned the value of 1 , those between $15 \%$ and $29.9 \%$ a value of 2 , and those $\geqslant 30 \%$ a value of 3 . Repeat measures analysis of variance with Tukey-Kramer post-test comparison was then used to assess the differences between the CHD risk scores from the three different CHD risk prediction methods.

\section{RESULTS}

During the study period, 1246 requests were received. The following requests were excluded: 51 due to inadequate clinical data, 10 because serum samples were erroneously discarded before lipid measurement, 10 because they were repeat requests, 67 on subjects aged $>70$ years because the new Sheffield table uses this as its upper age limit, and seven with LVH because LVH is not accommodated for in the JBS chart. Therefore, data from 1102 requests were evaluated (table 1).

The new Sheffield table CHD risk scores were higher $(\mathrm{p}<0.001)$ in comparison with the calculated CHD risk and modified JBS chart. The JBS chart risk scores were lower $(\mathrm{p}<0.05)$ than calculated CHD risk scores. The new Sheffield table and modified JBS chart performed equally well; both had high diagnostic sensitivity and specificity at 15\% and 30\% risk (table 2 ). The new Sheffield table identified all 38 subjects, with a 10 year CHD risk of $\geqslant 30 \%$ and serum total cholesterol $\geqslant 5.0 \mathrm{mmol} / \mathrm{l}$, requiring statin treatment; whereas the JBS chart failed to identify three of these subjects. The new Sheffield table misclassified 17 patients, with a 10 year CHD risk $<30 \%$ as requiring statin treatment ( 10 year CHD risk of $26.7 \%-29.8 \%)$; whereas the modified JBS chart did not misclassify any patients.

In comparison with the other methods of risk assessment, using the new Sheffield table as a screening tool to assess which subjects required measurement of serum lipids would have led to a $13.4 \%$ reduction in requests for serum lipid measurements ( $16.4 \%$ in women and $10.1 \%$ in men) without loss of diagnostic efficacy.

\section{DISCUSSION}

This study, the largest to date, confirms the high diagnostic sensitivity and specificity of the modified JBS chart and new Sheffield table ${ }^{57}$ and therefore their suitability for use in primary care. The high and equable diagnostic performance of

Table 1 Characteristics of subjects; results are mean (SD) or per cent

\begin{tabular}{lll}
\hline & Male $(\mathrm{n}=583)$ & Female $(\mathrm{n}=519)$ \\
\hline Age (years) & $52.1(10.2)$ & $55.3(9.5)$ \\
Systolic BP (mm Hg) & $143.1(19.3)$ & $142.9(20.1)$ \\
Total cholesterol (mmol/l) & $5.71(1.08)$ & $5.97(1.09)$ \\
HDL cholesterol (HDL) (mmol/I) & $1.23(0.37)$ & $1.42(0.38)$ \\
Total cholesterol: HDL cholesterol & $4.98(1.53)$ & $4.47(1.35)$ \\
Subjects with diabetes (\%) & $78(13.3)$ & $62(11.9)$ \\
Current smokers (\%) & $155(26.6)$ & $111(21.3)$ \\
Subjects with systolic BP $\geqslant 160 \mathrm{~mm} \mathrm{Hg} \mathrm{( \% )}$ & $131(22.5)$ & $120(23.1)$ \\
Subjects with systolic BP 140-159 mm Hg (\%) & $223(38.3)$ & $215(41.4)$ \\
10 year CHD risk & $14.4(10.1)$ & $9.7(6.7)$ \\
Subjects with 10 year CHD risk $\geqslant 30 \%(\%)$ & $38(6.5)$ & $10.8)$ \\
Subjects with 10 year CHD risk 15\%-29\% (\%) & $203(34.8)$ & \\
\hline BP, blood pressure; HDL, high density lipoprotein. &
\end{tabular}

Table 2 Diagnostic performance of the new Sheffield table and modified JBS chart

\begin{tabular}{lll}
\hline & Modified JBS chart & New Sheffield table \\
\hline At 15\% risk & $93.6(90.4$ to 96.0$)$ & $91.6(86.7$ to 95.1$)$ \\
Sensitivity (\%) & $94.7(92.6$ to 96.1$)$ & $93.8(91.1$ to 97.9$)$ \\
Specificity (\%) & $94.2(92.7$ to 95.6$)$ & $93.1(91.4$ to 94.6$)$ \\
Efficiency (\%) & $88.7(84.7$ to 91.9$)$ & $87.1(81.4$ to 91.4$)$ \\
PV +ve result (\%) & $97.0(95.5$ to 98.1$)$ & $96.1(93.8$ to 97.7$)$ \\
PV -ve result (\%) & $90.5(75.6$ to 97.4$)$ & $95.2(82.8$ to 99.4$)$ \\
At 30\% risk & $100(99.7$ to 100$)$ & $97.9(96.8$ to 98.7$)$ \\
Sensitivity (\%) & $99.6(99.0$ to 99.9$)$ & $97.8(94.6$ to 97.0$)$ \\
Specificity (\%) & $100(99.9$ to 100$)$ & $64.5(46.3$ to 77.8$)$ \\
Efficiency (\%) & $99.6(99.0$ to 99.9$)$ & $99.8(99.3$ to 100$)$ \\
PV +ve result (\%) &
\end{tabular}


both charts suggests that the approximations regarding age and blood pressure do not significantly affect their accuracy. The results reported in this study are consistent with reports suggesting that tables and charts which incorporate individual HDL cholesterol concentrations have overcome the inaccuracies of tables and charts which used a population average HDL cholesterol value..$^{9-11}$

The National Service Framework recommends use of the JBS chart but states that new Sheffield table, European coronary risk chart, and New Zealand cardiovascular risk prediction charts are acceptable for assessing CHD risk. ${ }^{1}$ We chose to evaluate the new Sheffield table and modified JBS chart as they are widely available, commonly used, and compatible with National Service Framework guidelines. The European coronary risk chart uses average HDL cholesterol values and is therefore inherently inaccurate ${ }^{7}$ and should not be used. The New Zealand cardiovascular risk prediction charts calculate cardiovascular risk rather than CHD risk and are therefore less compatible with National Service Framework and UK guidelines which offer recommendations for management of CHD risk. ${ }^{16}$

It is desirable that CHD risk tables and charts should have high sensitivity (no false negatives) to identify the maximum of high risk patients who would benefit most from drug treatment. It is also important that they have high specificity (no false positives) so that they do not falsely label individuals as being high risk and requiring drug treatment. The new Sheffield table was more sensitive (fewer false negatives) but less specific (more false positives) at 30\% risk, and marginally less sensitive and specific at $15 \%$ risk in comparison with the modified JBS chart. Our results are similar but not identical to a recent smaller study reporting that the modified JBS chart should be method of choice for predicting CHD risk from tables. ${ }^{7}$ We, however, conclude that since the tables perform comparatively well the choice of chart lies in ease of use and their potential impact on healthcare costs.

We found the compact tabular layout of the Sheffield table easier to use but others have reported a preference for the graphic displays of the modified JBS chart. ${ }^{12}$ An advantage of the graphic display of modified JBS chart is that it provides graded risk denoted as three differently coloured zones $(<15 \%, \geqslant 15 \%$ to $<30 \%$, and $\geqslant 30 \%)$, unlike the new Sheffield table that provides cut off values for risk at $15 \%$ and $30 \%$. Therefore the modified JBS chart (unlike the new Sheffield table) can, for example, indicate the difference between $16 \%$ and $29 \%$ risk and this may have patient management implications.

In this study, the lower specificity at $30 \%$ risk of the new Sheffield tables in comparison with the modified JBS chart, necessarily increases "false positives" and therefore total prescribing statin costs. This, however, has to be offset against potential cardiovascular morbidity costs of the modified JBS chart in failing to identify and treat very risk high subjects. A not widely appreciated advantage of the new Sheffield table over all other assessment methods is its ability to screen which subjects do not require a blood test for measurement of total and HDL cholesterol concentrations. ${ }^{5}$ This could result in potential savings in healthcare resources by reducing laboratory costs, clinical costs (fewer revisits), and be seen as an improvement in patient care by reducing the number of unnecessary venepunctures and surgery visits. Even in this study targeted to subjects with potentially increased CHD risk, the new Sheffield table would have led to a $13.4 \%$ fewer lipid measurements in comparison with the modified JBS chart and computer program without loss of diagnostic efficacy.

An alternative to tables and charts is an individualised CHD risk score calculated on personal computers which give a more precise prediction of CHD risk. ${ }^{16}{ }^{13}$ In offering more patient interaction by illustrating the benefits of modifying risk (for example, stopping smoking), computer programs may be preferable to tables in managing cardiac risk. ${ }^{13}$ A potential

\section{Learning points ${ }^{1}$}

- Management of borderline hypertension, hyperlipidaemia and use of aspirin in the primary prevention of cardiovascular disease is based on the individual's predicted coronary heart disease risk.

- CHD risk assessment is based on the Framingham model. Although validated for the British population, it may underestimate risk in familial hyperlipidaemias, certain ethnic groups, and those with a family history of premature CHD.

- Treat sustained borderline hypertension (systolic blood pressure $\geqslant 140$ to $<160 \mathrm{~mm} \mathrm{Hg}$ and/or diastolic blood pressure $\geqslant 90$ to $<100 \mathrm{~mm} \mathrm{Hg}$ ) in the presence of: target organ damage; diabetes mellitus; and 10 year CHD risk $\geqslant 15 \%$.

- Prescribe, if not contraindicated, $75 \mathrm{mg}$ aspirin in hypertensive patients $>50$ years after adequate control of blood pressure $(<150 / 90 \mathrm{~mm} \mathrm{Hg})$ in the presence of: target organ damage; diabetes mellitus; and 10 year CHD risk $\geqslant 15 \%$.

- Prescribe statins if 10 year CHD risk is $\geqslant 30 \%$ and serum total cholesterol is persistently $\geqslant 5.0 \mathrm{mmol} / \mathrm{l}$.

disadvantage of surgery based computer calculated CHD risk is that the practitioner is required to collate and enter clinical and biochemical data. An alternative is a laboratory based CHD risk calculation, ${ }^{7114}$ which has the added advantages of easily disseminating and implementing locally and nationally revised guidelines. Although computer programs give a more precise prediction of risk than charts, other factors including familiarity, speed, and ease of use during consultation are also important and paper based charts may still have a useful role in assessing CHD risk.

Prediction of individualised CHD risk without resorting to a risk assessment tool is often erroneous with medical practitioners either under-estimating or over-estimating risk, ${ }^{15}{ }^{16}$ and this may lead to erroneous patient management decisions. ${ }^{17}$ Implementing systematic CHD risk assessment in primary care can be facilitated by cardiovascular disease registers to identify high risk individuals. The likely increase in workload may be a cause for concern, ${ }^{18}$ but CHD risk assessment is a requirement of the National Service Framework. ${ }^{1}$

In conclusion, the new Sheffield table and modified JBS chart perform equally well in predicting CHD risk for primary care patients. Since assessing an individual's CHD risk is not intuitive we suggest that health practitioners should choose a risk assessment tool (the new Sheffield table or modified JBS chart or computer program) with which they are comfortable and then use it.

\section{ACKNOWLEDGEMENTS}

We thank Dr A F Jones for statistical advice and Mrs Rabindranath for secretarial assistance.

\section{Authors' affiliations}

K S Rabindranath, Department of Medicine, New Cross Hospital, Wolverhampton, UK

N R Anderson, R Gama, M R Holland, Department of Clinical Chemistry

\section{REFERENCES}

1 Department of Health. The National Service Framework of coronary heart disease. Modern standards and service models. London: DoH, 2000.

2 Anderson KM, Odell PM, Wilson PWF, et al. Cardiovascular disease risk profiles. Am Heart J 1991;121:293-8.

3 Ramachandran S, French JM, Vanderpump MPJ, et al. Using the Framingham model to predict heart disease in the United Kingdom: retrospective study. BN 2000;320:676-7.

4 Messerli FH, Mittler BS. Framingham at 50. Lancet 1998;352:1006. 
5 Wallis EJ, Ramsay LE, UI Haq I, et al. Coronary and cardiovascular risk estimation for primary prevention: validation of a new Sheffield table in the 1995 Scottish health survey population. BM 2000;320:671-85.

6 British Cardiac Society, British Hyperlipidaemia Association, British Hypertension Society, British Diabetic Association. Joint British recommendations on prevention of coronary disease in clinical practice. BM 2000;320:705-8.

7 Jones AF, Walker J, Jewkes C, et al. Comparative accuracy of cardiovascular risk prediction methods in general practice patients. Heart 2001;85:37-43.

8 Ramsay LE, Williams B, Johnston GD, et al. British Hypertension Society guidelines for hypertension management 1999: summary. BM 1999;319:630-5.

9 Betteridge J, Shepherd J, Thompson G. Sheffield tables have shortcomings. Lancet 1997;350:1174-5.

10 Haq IU, Ramsay LE, Jackson PR, et al. Prediction of coronary risk for primary prevention of coronary heart disease: a comparison of methods. Q J Med 1999;92:379-85.

11 Bayly GR, Bartlett WA, Davies PH, et al. Laboratory-based calculation of coronary heart disease in a hospital diabetes clinic. Diabet Med 1999; 16:697-701
12 Isles CG, Ritchie LD, Murchie P, et al. Risk assessment in primary prevention of coronary heart disease: randomised comparison of three scoring methods. BM 2000;320:690-1.

13 Hingorani AD, Vallance P. A simple computer program for guiding management of cardiovascular risk factors and prescribing. BM 1999;318:101-5.

14 Gerbhiwot T, Jones AF, Bartlett WA, et al. Laboratory-based calculation of coronary heart disease risk. Clin Chem 2001;47:589-91.

15 Grover SA, Lowensteyn I, Esrey KL, et al. Do doctors accurately assess coronary risk in their patients? Preliminary results of the coronary health assessment study. BM 1995;310:975-8.

16 Montgomery AA, Fahey T, MacKintosh C, et al. Estimation of cardiovascular risk in hypertensive patients in primary care. $\mathrm{Br} J \mathrm{Gen}$ Pract 2000;50:127-8.

17 Ramachandran S, Labib MH. Hyperlipidaemia and primary prevention of coronary heart disease: are the right patients being treated? J Cardiovasc Risk 2000; 7:245-9.

18 Pringle $M$. Preventing ischaemic heart disease in one general practice: from one patient, through clinical audit, needs assessment, and commissioning into quality improvement. BN 1998;317:1120-3. 